\title{
Development of the BAND-GEM detector solution for SANS experiments
}

\author{
Gabriele Croci, $^{a, b, d, 1}$, Andrea Murarob $^{b}$, Enrico Perelli Cippo ${ }^{b}$, Giorgia Albani ${ }^{a}$, Jens \\ Birch $^{e}$, Giovanni Grosso ${ }^{b}$, Carina Höglund ${ }^{\mathrm{e}, \mathrm{g}}$, Lars Hultman ${ }^{\mathrm{e}}$, Fabrizio Murtas ${ }^{\mathrm{c}}$, \\ Marica Rebai $^{\mathrm{a}, \mathrm{b}}$, Marco Tardocchi $^{\mathrm{b}}$, Isabel Jansa Llamas ${ }^{\mathrm{h}}$ and Giuseppe Gorini ${ }^{\mathrm{a}, \mathrm{b}, \mathrm{d}}$ \\ a Dipartimento di Fisica “G. Occhialini”, Università degli Studi di Milano-Bicocca, Milano, 20125, \\ Italy \\ ${ }^{b}$ Istituto di Fisica del Plasma “P. Caldirola”, Associazione EURATOM-ENEA/CNR, Milano, 20125, \\ Italy \\ ${ }^{c}$ Istituto Nazionale di Fisica Nucleare, Laboratori Nazionali di Frascati, Frascati, 0044, Italy \\ ${ }^{d}$ Istituto Nazionale di Fisica Nucleare, Sezione di Milano Bicocca, Milano, 20125, Italy \\ ${ }^{e}$ Dept. of Physics, Chemistry and Biology (IFM), Linköping University, SE-581 83 Linköping, Sweden \\ ${ }^{f}$ Institute for Energy Technology, Instituttveien 18, NO-2007 Kjeller, Norway \\ ${ }^{g}$ European Spallation Source ESS AB, Tunavägen 24, 22363 Lund, Sweden \\ ${ }^{h}$ Institute for Energy Technology, Instituttveien 18, NO-2007 Kjeller, Norway
}

New high count rate detectors are needed for future spallation neutron sources where large areas and high efficiency $(>50 \%)$ detectors are envisaged. In this framework, GEM is one of the explored detector technologies since they feature good spatial resolution $(<0.5 \mathrm{~cm})$ and timing properties, have excellent rate capability $(\mathrm{MHz} / \mathrm{mm} 2)$ and can cover large areas (some $\mathrm{m} 2)$ at low cost. In the BAND-GEM (Boron Array Neutron Detector) approach a 3D geometry for the neutron converter cathode was developed that is expected to provide an efficiency $>20 \%$ in the wavelength range of interest for SANS instruments. A system of thin lamellas $(250 \mathrm{~m})$ of dielectric material coated with 1 m layer of ${ }^{10} \mathrm{~B}_{4} \mathrm{C}$ has been built and positioned in the first detector gap, orthogonally to the cathode. By tilting the lamellas system with respect to the beam, there is a significant increase of effective thickness of the borated material crossed by the neutrons. As a consequence, both interaction probability and detection efficiency are increased. This paper presents the results of the performance of the first prototype of BAND-GEM detector in terms of efficiency (as a function of tilting angle). you can write the abstract for your paper.

5th International Conference on Micro-Pattern Gas Detectors (MPGD2017)

22-26 May, 2017

Philadelphia, USA

\footnotetext{
${ }^{1}$ Speaker: gabriele.croci@unimib.it 


\section{Introduction}

Neutron diffraction and scattering experiments using thermal neutrons are the core activity at spallation neutron sources. Detection systems nowadays make large use of ${ }^{3} \mathrm{He}$-based gaseous detectors that give the possibility to cover large areas $\left(\mathrm{m}^{2}\right)$ and have an intrinsic efficiency to thermal neutrons higher than $80 \%$. ${ }^{3} \mathrm{He}$ shortage and its massive use in applications linked to homeland security have determined an exponential rise of its price, preventing its use for research applications including future neutron sources such as the European Spallation Source (ESS [1]). In the case of ESS, besides the need for replacing ${ }^{3} \mathrm{He}$, it is essential to develop highrate neutron detectors able to fully exploit the increase of neutron flux of ESS relative to present neutron sources. The situation calls for the development of large area and high-rate neutron detectors that do not use ${ }^{3} \mathrm{He}$ and have comparable detection efficiency with a price ideally not exceeding $100 \mathrm{k} € / \mathrm{m}^{2}$ and a space resolution 1- $10 \mathrm{~mm}$. This paper describes a new detector based on the GEM technology [2] that is able to detect beams at high rate $\left(>1 \mathrm{MHz} / \mathrm{mm}^{2}\right)$ and gives the possibility to cover large areas at low cost. Although GEM-based detectors are mostly used to detect charged particles, they can be adapted (typically by using a customized cathode configuration), to detect neutral particles, such as neutrons and photons (where other detectors are usually exploited [3]). Several GEM detectors for photon, fast and thermal neutron detection have been realized during the last years [4]-[23].

The BAND-GEM detector described in this paper aims at increasing the thermal neutron detection efficiency with respect to single layer boron based detectors. The strategy that has been adopted consists in the realization of a cathode with 3-dimensional structure similarly to what is described in [24], [25].

\section{BAND-GEM Detector construction}

The Boron Array Neutron Detector based on the GEM technology (BAND-GEM) described in this paper is constituted by a Triple GEM detector equipped with a 3D converter cathode. This cathode is constituted by a system of thin lamellas of dielectric material $\left(\mathrm{Al}_{2} \mathrm{O}_{3}\right)$ coated with a 1 m layer of enriched $\mathrm{B}_{4} \mathrm{C}$ on both sides; the lamellas system is positioned in the first detector gap orthogonally to the planar cathode (see Figure 1). If the full detector is inclined by few degrees (less than $10^{\circ}$ ) with respect to the incoming beam, neutrons are forced to cross the boron layers at grazing angles. As a consequence, interaction probability, as well as the detection efficiency, is augmented while keeping the beam perturbation small (due to the reduced volume of non-active material). Moreover, conservation of energy and momentum for the $n\left({ }^{10} \mathrm{~B},{ }^{7} \mathrm{Li}\right) \alpha$ occurring in the layer implies that the two charged particles $\left({ }^{7} \mathrm{Li}\right.$ and $\left.\alpha\right)$ are emitted back to back with kinetic energies in the order of $1 \mathrm{MeV}$. It means that at least one of the (charged) reaction products is likely to be able to leave the ${ }^{10} \mathrm{~B}_{4} \mathrm{C}$ layer and thus be revealed. The gas mixture used in the detector is $\mathrm{Ar} / \mathrm{CO}_{2} 70 \% / 30 \%$ and it is characterized by a mean work function of about $27 \mathrm{eV}$. In order to get a signal the primary electrons created either by alphas or $7 \mathrm{Li}$ ions must be able to move inside the lamella system and reach the Triple GEM structure where they are multiplied. In order to extract the primary charge, each lamella is segmented into strips each kept at a different voltage: this allows generating a drift field in the system that is as uniform as possible. Since the ${ }^{10} \mathrm{~B}_{4} \mathrm{C}$ deposit is slightly conductive, this field can be generated by dividing each lamella in 15 strips (all covered by the converter material) and by applying a 
different potential to each strip. A particular ${ }^{10} \mathrm{~B}_{4} \mathrm{C}$ coating procedure, performed at the Linkoping University, was studied in order to coat only the strips and not the full lamella plate: this was possible thanks to the realization of especially designed supports covering the surface that must not be coated. The detailed characterization of the coating is described in [20]. The lamella system can be thus considered as a field cage and the potential to each strip is given through an external voltage divider that has the two ends connected between the planar cathode and the top electrode of the first GEM foil (GEM 1 Top).

A detailed engineering design has been performed in order to realize the full system guaranteeing that each lamella is correctly sustained and each strip gets the right potential.

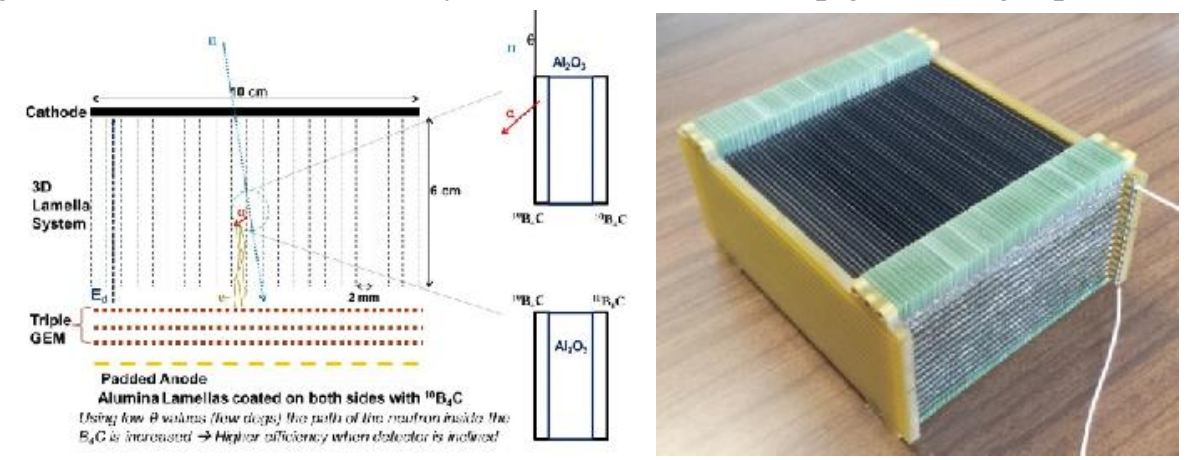

Figure 1. Left Detector schematics and principle of operation; Right: picture of the 3D cathode

In order to extract the primary charge, each lamella is segmented into strips each kept at a different voltage: this allows generating a drift field in the system that is as uniform as possible. Since the ${ }^{10} \mathrm{~B}_{4} \mathrm{C}$ deposit is slightly conductive, this field can be generated by dividing each lamella in 15 strips (all covered by the converter material) and by applying a different potential to each strip. A particular ${ }^{10} \mathrm{~B}_{4} \mathrm{C}$ coating procedure, performed at the Linkoping University [22], was studied in order to coat only the strips and not the full lamella plate: this was possible thanks to the realization of especially designed supports covering the surface that must not be coated. The lamella system can be thus considered as a field cage and the potential to each strip is given through an external voltage divider that has the two ends connected between the planar cathode and the top electrode of the first GEM foil (GEM 1 Top).

A detailed engineering design has been performed in order to realize the full system guaranteeing that each lamella is correctly sustained and each strip gets the right potential.

\section{Detector construction and characterization on a neutron beam}

Figure 2 shows a series of picture showing the detector assembly into its containing box. The triple GEM box is closed on top of the lamella box and the appropriate electric contacts are realized. This BAND-GEM detector is equipped with a padded anode composed by 128 pads each with a dimension of $12 \times 6 \mathrm{~mm}^{2}$. Each signal of each pad is routed to a different channel of one CARIOCA [26] chip whose connector is located on the back of the read-out anode board. 


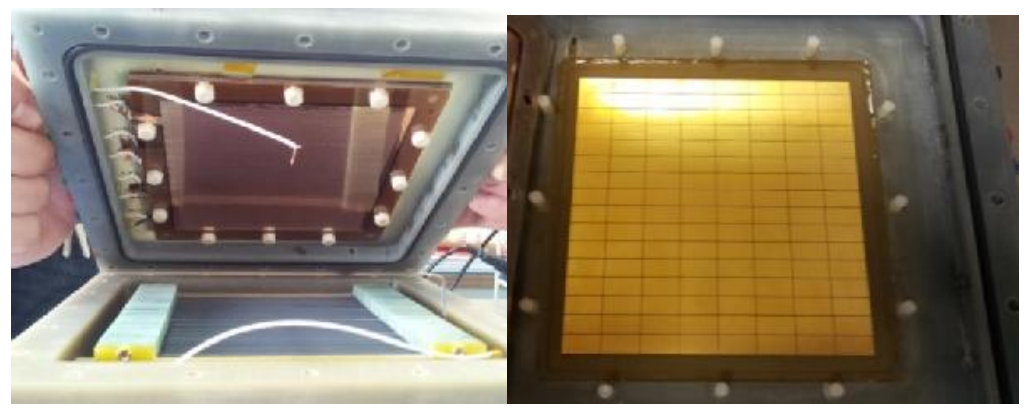

Figure 2: Detector Assembly

The detector was tested at the R2D2 (JEEP II reactor Kjeller, Norway) which features $1.54 \AA$ neutrons with a flux of about $10^{5} \mathrm{n} \mathrm{cm}^{2} \mathrm{~s}^{-1}$. The BAND-GEM detector prototype was positioned in the beam immediately after two collimation slits. The lamella system resistor chain was biased by connecting its terminals one to the Cathode voltage and the other to the GEM 1 Top voltage using the HVGEM module. Following this configuration the maximum achievable potential difference on the system is about $1.5 \mathrm{kV}$. The gas flow used during all the measurement is $\mathrm{Ar} / \mathrm{Co}_{2} 70 \% / 30 \%$ with a flow of about $51 / \mathrm{h}$. The beam line is provided with two ${ }^{3} \mathrm{He}$ tubes that are used as flux monitors. The first one is located before two collimating slits, has an efficiency of about $10^{-4}$ at $1.54 \AA$. The other one has an efficiency of about $80 \%$ at $1.54 \AA$ and was used as reference detector during the efficiency measurements. The gas gain of Triple GEM based detectors exponentially depends on the sum of the potential differences applied to the three GEM foils ( vGEM1 $\left.+V_{\mathrm{GEM} 2}+\mathrm{V}_{\mathrm{GEM} 3}=\mathrm{V}_{\mathrm{GEM}}\right)$. Due to the loss of primary charge in the lamella system, it is fundamental to understand which is the gas gain needed to obtain a certain detection efficiency. The beam was collimated and had a nominal 2D profile of $5 \mathrm{~mm} \mathrm{x}$ $5 \mathrm{~mm}$. Such a collimated beam was necessary in order to study local effects on the lamella system. The GEM detector efficiency was measured as a function of the effective gain by varying $\mathrm{V}_{\mathrm{GEM}}$ and the results are shown in Figure 3 . The counting rate is an increasing function of VGEM and, as expected, the detector starts to detect thermal neutrons at a voltage $\mathrm{V}_{\mathrm{GEM}}=$ $810 \mathrm{~V}$ (i.e. gain of about 50). In order to increase the counting rate, the voltage was pushed up to $1140 \mathrm{~V}$ and a variation of the curve slope is observed at $\mathrm{V}_{\mathrm{GEM}}=920 \mathrm{~V}$. The gain corresponding to this voltage (i.e. 4000) was interpreted as the gain where the detector starts to be sensitive to gamma rays. As working point a $\mathrm{V}_{\mathrm{GEM}}=980 \mathrm{~V}$ was selected.
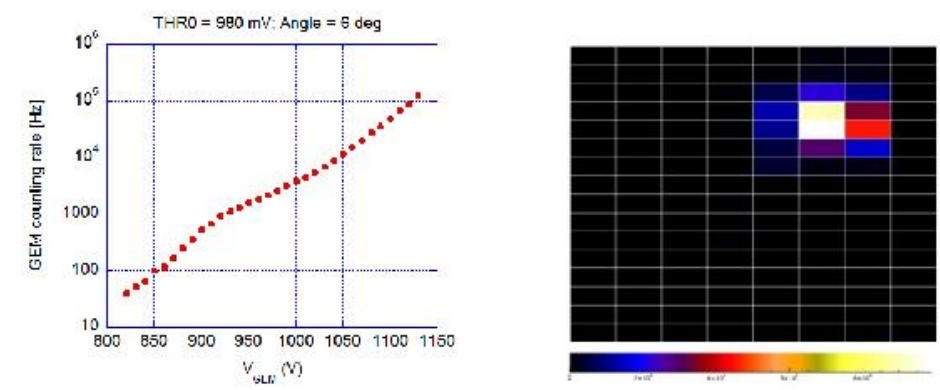

Figure 3: Left: VGEM scan used to determine the detector working point; right: 2D neutron beam profile reconstruction. The detector was tilted by 7 .

By using this GEM voltage and by tilting the detector by 7 degrees with respect to the collimated incoming beam, the beam profile was reconstructed, as shown in Figure 3. The counting rate of the detector was also measured as a function of the tilting angle (i.e. the angle between the beam direction and the lamella system) in order to verify the principle of operation of the device itself (see Figure 1).

Figure 4 shows the result the angular scan measurement. The BAND-GEM counting rate oscillates with different amplitudes up to $10^{\circ}$ where it stabilizes on a plateau. The reasons for 
these oscillations are linked to the geometrical parameters of the lamella system. In order to understand if the observed oscillation effect was really due to different absorption of the detector, the same measurement was repeated by putting the ${ }^{3} \mathrm{He}$ reference tube behind the BAND-GEM. The BAND-GEM was kept off and its signal cables and electronics were removed. Figure 10right shows the ${ }^{3} \mathrm{He}$ counting rate as a function of the GEM rotation angle. It is possible to appreciate that the $3 \mathrm{He}$ counting rate is complementary to what has been measured using the BAND-GEM: if the BAND-GEM shows a deep, the ${ }^{3} \mathrm{He}$ shows a peak for the same tilting angle. For the BAND-GEM a ratio between the deep $\left(4^{\circ}\right)$ to plateau $\left(>11^{\circ}\right)$ of 3.1 was measured. On the other hand, the ${ }^{3} \mathrm{He}$ show a peak $\left(4^{\circ}\right)$ to plateau $\left(>11^{\circ}\right)$ ratio of only 2 . The difference between these two values can be ascribed to the extraction efficiency of the BANDGEM lamella system since not all the converted neutrons are able to generate a signal over the threshold.
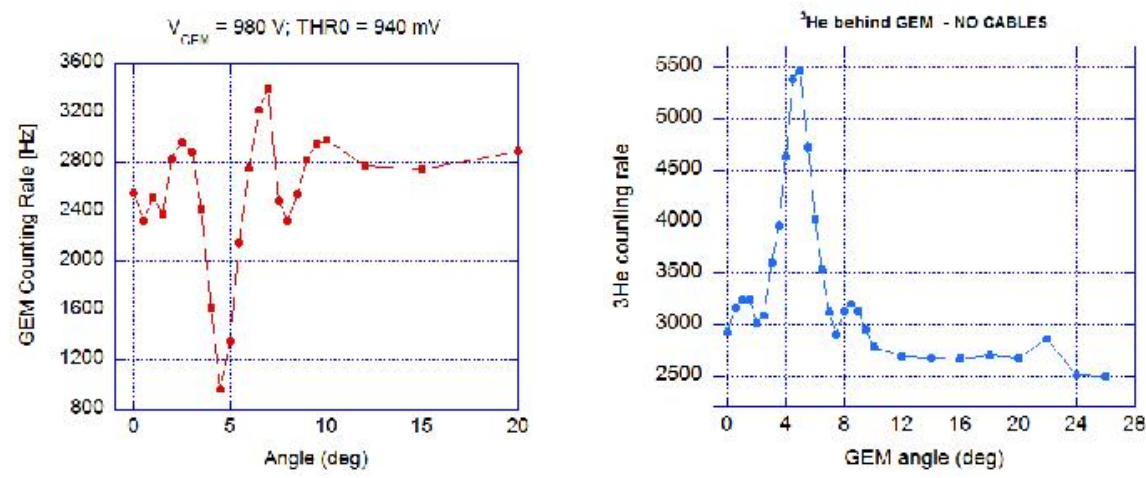

Figure 4: Left: GEM counting rate as a function of the tilting angle; Right: $3 \mathrm{He}$ counting rate as a function of GEM tilting angle. The $3 \mathrm{He}$ tube is positioned behind the GEM detector

At an angle of $10^{\circ}$ an efficiency $\varepsilon$ of about $16 \%$ was obtained by comparison with the ${ }^{3} \mathrm{He}$ tube.s.

\section{Conclusions and future perspectives}

A first prototype of BAND-GEM thermal neutron detector for SANS experiments for ESS has been successfully realized and tested. This detector represents a first step towards the realization of a higher efficiency GEM-based thermal neutron detector that can represent a serious alternative to standard ${ }^{3} \mathrm{He}$ detectors for SANS measurements.

\section{References}

[1] F. Sauli, Nucl. Instrum. Meth. A, 386, 531 (1997).

[2] M. Alexeev et Al, "The quest for a third generation of gaseous photon detectors for Cherenkov imaging counters", Nuclear Instruments and Methods in Physics Research Section A: Accelerators, Spectrometers, Detectors and Associated Equipment, Volume 610, Issue 1, 21 October 2009, Pages 174-177

[3] M. Rebai et Al, "Pixelated Single-crystal Diamond Detector for fast neutron measurements" 2015 JINST 10 C03016

[4] M. Alexeev et Al, "THGEM based photon detector for Cherenkov imaging applications", Nuclear Instruments and Methods in Physics Research Section A: Accelerators, Spectrometers, Detectors and Associated Equipment, Volume 617, Issues 1-3, 11 May 2010-21 May 2010, Pages 396-397;

[5] M. Alexeev et Al, "Development of a THGEM-based photon detector for Cherenkov Imaging counters ", 2010 JINST 5 P03009 
[6] M. Alfonsi et Al, "Simulation of the dielectric charging-up effect in a GEM detector ", Nuclear Instruments and Methods in Physics Research Section A, Volume 671, 11 April 2012, Pages 6-9

[7] M. Alexeev et Al, "Micropattern gaseous photon detectors for Cherenkov imaging counters", Nuclear Instruments and Methods in Physics Research Section A: Accelerators, Spectrometers, Detectors and Associated Equipment, Volume 623, Issue 1, 1 November 2010, Pages 129-131

[8] M. Rebai et Al, "A new diagnostic concept for high power deuterium beams", Review of Scientific Instruments, 83, 02B721 (2012)

[9] R. Pasqualotto et Al, "Diagnostics of the ITER neutral beam test facility”, Review of Scientific Instruments, 83, 02B103 (2012)

[10] G. Croci et Al, "nGEM neutron diagnostic concept for high power deuterium beams”, 2012 JINST $7 \mathrm{C} 03010$

[11] F. Murtas et Al, "Triple GEM gas detectors as real time fast neutron beam monitors for spallation neutron sources”, 2012 JINST 7 P07021

[12] G. Croci et $A l$, "nGEM fast neutron detectors for beam diagnostics", Nuclear Instruments and Methods in Physics Research Section A, Volume 720, 21 August 2013, Pages 144-148

[13] G. Croci et $A l$, "Discharge probability measurement of a Triple GEM detector irradiated with neutrons”, NIM A, Volume 712, 1 June 2013, Pages 108-112

[14] G. Croci et $A l$, "Measurements of $\gamma$-ray sensitivity of a GEM based detector using a coincidence technique", 2013 JINST 8 P04006

[15] G. Croci et Al, "GEM-Based thermal neutron beam monitors for spallation sources", Nuclear Instruments and Methods in Physics Research Section A, Volume 732, 21 December 2013, Pages $217-220$

[16] G. Croci et Al, "Diffraction measurements with a boron-based GEM neutron detector", EPL, 107 (2014) 12001

[17] G. Croci et Al, "Characterization of a thermal neutron beam monitor based on gas electron multiplier technology”, Prog. Theor. Exp. Phys. (2014) 083H01

[18] E. Perelli Cippo, G. Croci et Al, "A GEM-based thermal neutron detector for high counting rate applications, 2015 JINST 10 P10003

[19] G. Albani, G. Croci et Al, "Neutron beam imaging with GEM detectors” 2015 JINST 10 C04040

[20] G. Croci et Al, “GEM-based detectors for thermal and fast neutrons”, EPJP (2015) 130:118

[21] A. Muraro et Al, "Performance of the full size nGEM detector for the SPIDER experiment", NIMA, Volume 813, 21 March 2016, Pages 147-152

[22] G. Croci et Al, "GEM-based detectors for thermal and fast neutrons", EPJP (2015) 130:118

[23] A. Muraro, G. Croci et Al, "Status of the CNESM diagnostic for SPIDER", Fusion Engineering and Design, Volumes 96-97, October 2015, Pages 311-314

[24] A. Muraro et Al, Neutron radiography as a non-destructive method for diagnosing neutron converters for advanced thermal neutron detectors”, 2016 JINST 11 C03033

[25] G. Albani et Al, Evolution in boron-based GEM detectors for diffraction measurements: from planar to 3D converters, Meas. Sci. Technol. 27115902

[26] W Bonivento et al, Nucl. Instrum. Meth. A, 491, 233 (2002)

[27] A. Muraro et Al, "Performance of the high efficiency BAND-GEM detector" Prog. Theor. Exp. Phys. 2018, 023H01 (17 pages) DOI: 10.1093/ptep/pty005 